\title{
Conceptual bases, elements of methodology and narrative aspects resulted from direct clinical experience - afferent to pulmonary rehabilitation in post COVID-19 patients
}

\author{
Liliana Padure ${ }^{1}$, Cristina Popescu ${ }^{3}$, Aura Spinu ${ }^{3,4}$, Cristina Daia ${ }^{3,4}$, Andra Pintilie ${ }^{1}$, \\ Gelu Onose ${ }^{3,4}$, Adrian Streinu Cercel ${ }^{2,4}$ \\ 1"Dr. Nicolae Robanescu” National Clinical Teaching Centre for Pediatric Neuropsychomotor Rehabilitation, \\ Bucharest, Romania \\ 2"Prof. Dr. Matei Bals" National Institute of Infectious Diseases, Bucharest, Romania \\ 3"Bagdasar-Arseni" Teaching Emergency Hospital, Bucharest, Romania \\ 4"Carol Davila" University of Medicine and Pharmacy, Bucharest, Romania
}

\begin{abstract}
Introduction. Coronavirus disease 2019 (COVID-19) has been declared as a new illness in December 2019 in Hubei Province, China and considered a pandemic by the WHO Director General's opening remarks at the related media briefing in March 2020. This illness is caused by a virus called Severe Acute Respiratory Syndrome Coronavirus 2 (SARS-CoV-2). COVID-19 involves multiple organ impairments and lung injury is one of the most common lesions of this disease. Although it has been studied for nine months, our understanding of this disease is still incomplete, including in terms of sequelae and long-term outcomes.

A large number of patients are affected by the COVID-19 pandemic but only in some patients the disease has progressed rapidly to respiratory failure requiring hospitalization or treatment in intensive care units. The most common complications noticed in severe cases is acute respiratory distress syndrome with a consecutive respiratory failure.

Materials and method. The detailed evaluation of the patient, the methodological and fundamental elements of the pulmonary rehabilitation adapted to the patient are interventions which have an extremely important role and application in COVID-19 treatment, in mitigating dyspnoea symptoms, anxiety relief, reducing other possible complications, minimizing disability, maintaining functionality, all in order to increase the patient quality of life. As for October 2020 and still ongoing, a number of approximately fifty patients from both the Intensive Care Unit and clinical wards of "Prof. Dr. Matei Bals" National Institute of Infectious Disease have been evaluated by the physician and underwent a physical therapy program, assisted by the physiotherapist.

Results. Lung rehabilitation should be considered when possible and safe during treatment of patients with COVID-19 and may include rehabilitative nursing, specific respiratory kinesiotherapy and for increasing general effort capacity and mobility (see further for methodological details).

From the physician's point of view, it is extremely important to carefully conduct the anamnestic process and evaluation, to adjust the program to each individual, to monitor oxygen saturation, blood pressure levels, pulse and temperature throughout the exercises. Every increment or decrement of these parameters, onset of cough or fatigability, must end the rehabilitation process.

Conclusions. Like for all the chronic pulmonary patients in the cases where COVID-19 resulted in respiratory sequels, specific and connected general physical and kinesiological rehabilitative interventions are necessary and useful. The methodology must be thoroughly case by case elaborated and applied considering also the epidemiologic related concerns.
\end{abstract}

Keywords: COVID-19, SARS-CoV-2, pulmonary rehabilitation

\section{BACKGROUND}

By the end of December 2019, a new outbreak, a potential severe acute respiratory disease (Severe
Acute Respiratory Syndrome - Coronavirus - 2 (SARS-CoV-2) causing COVID-19) emerged from Hubei Province, China, spilling rapidly all over the world and determining the World Health 
Organization (WHO) to declare it a public health emergency in January $2010(1,2,3)$, respectively a pandemic declared since March 2020 (4).

This new illness resulted to more than 500,000 deaths up to July 2020 and there is no pre- existing immunity or definitive treatment till date $(2,3,5)$.

Covid-19 shows a wide spectrum of symptoms, such as: fever, cough, malaise, headache, sore throat, dyspnoea, joints pain, diarrhoea, vomiting, loss of smell (anosmia), but also fatal pneumonia or multi-organ failure - with death occurring (most commonly) in the third week from the beginning of specific symptoms - and (also) some patients being asymptomatic $(1,3,6)$.

What scientific world knows by now, is that this virus is usually transmitted from one person to another, via aerosols $(6,7)$, and the incubation period ranges from 2 to 14 days. There is also a still open debate whether and in what conditions this virus can be transmitted by contacting contaminated surfaces (including its lasting infectiveness in there) (8). A feature of severe COVID-19 is related to patients comorbidities (i.e. diabetes mellitus - a major risk factor, leading as well to a higher mortality rate-, hypertension, autoimmune diseases, malignancy, chronic kidney disease, even obesity) or with an overreaction of the immune system including a severe citokyne storm and consequent activated coagulation. Older ages, immune-compromised patients and the presence of coagulopathies are at greatest risk of mortality $(3,6,9-12)$.

Distancing (physical spacing between individuals) is an evidence-based practice in preventing the transmission of the virus and in slowing the incidence of the disease (13-15). Wearing surgical masks is an efficient method to prevent the inhalation of large droplets but with a limited ability to stop the particles less than $5 \mu \mathrm{m}$ such as SARS-CoV-2 aerosols (16-18).

Being more contagious than Severe Acute Respiratory Syndrome (SARS) or Middle East Respiratory Syndrome (MERS), it is necessary a clear understanding of this virus pathology/ spreading all over the globe in order to obtain a reliable public health response to this pandemic (1).

\section{RESPIRATORY COMPLICATIONS OF COVID-19}

The impact of severe SARS-CoV-2 is still to be seen, but there are evidences proving that pulmonary symptomatology may persist months post their initial illness (19-21).

Almost all COVID-19 complications are related to pneumonia it causes. The mechanism throu- gh this new coronavirus causes lung damage is not completely known, but researchers mention by now: the citokynes storm, the drug-induced toxicity and hyperoxia induced by long term mechanical ventilation (22).

Many of the recovered patients experience dry cough or burning in their lungs. Computer tomography (CT) scan plays an important role in evaluation of COVID-19, although there is not a worldwide imaging consensus regarding the use of this method in the initial diagnosis or monitoring the evolution of diagnosed patients. SARS-CoV-2 affects both lungs, which in CT scan appear as "ground-glass opacities" with lower lobe predilection in all patients, which on the one hand may not heal and on the other, may cause permanent impairment (22-24).

Pneumothorax, usually occurring in very tall young men or in older patients with underlying lung disease, was described by the researchers of University of Cambridge in COVID-19 patients with neither of those risk factors. In some patients, the same study described an association between pneumotorax and pneumo-mediastinum, even among those who didn't need invasive ventilation (25).

The most common complications noticed in severe cases is acute respiratory distress syndrome with a consecutive respiratory failure (26-28). Not to be neglected that fatality rate among patients in critical state is about $49 \%$ (28).

A long term follow-up is the new challenge and is strongly recommended in these patients in order to early detect the lung sequels and to properly manage the pulmonary disfunction (29).

\section{PULMONARY REHABILITATION}

The target to be achieved in pulmonary rehabilitation is to relieve symptoms such as dyspnoea, anxiety, impairment, to diminish complications, all in order to increase the patient quality of life. "Pulmonary rehabilitation during the acute management of COVID-19 should be considered when possible and safe and may include nutrition, airway, posture, clearance technique, oxygen supplementation, breathing exercises, stretching, manual therapy, and physical activity" (30).

Being a disease yet without a cure or a vaccine to prevent, respiratory/ pulmonary rehabilitation plays an essential role both in early stages of post Covid patients, but also in survivors of severe COVID-19 (who may suffer, besides reduced lung function, myopathy, polyneuropathy, also of incre- 
ased anxiety and depression associated with their fear of survival) (30). Simultaneously, a preventive perspective is to be mentioned in order to diminish psychosocial problems, to improve the work performance and the functional status of individuals.

Pulmonary rehabilitation is based on personalized evaluation of every patient, including - without limiting though - exercise training, behaviour changes, education, to improve physical and psychological status of people suffering of respiratory impairments.

Recent studies showed that early mobilization in patients admitted in Intensive Care Units (ICU) due to acute respiratory failure necessiting mechanical support of respiration for more than 4 days is both safe and feasible, reducing supplementary costs and improving the quality of life $(31,32)$. At the same time, the physical deconditioning following a prolonged ICU stay, is responsible for many of the sequels related to the functional status of the individuals (32).

In mild cases of COVID-19 (meaning that pneumonia is not included) in outpatients, it is highly recommended the use of telemedicine. Using telehealth and pulmonary rehabilitation at home is proven to have the same outcomes with the medical center based programmes and additionally with a decreased risk of contamination of the health care workers. For these kind of patients, the rehabilitation programme includes: airway clearance techniques, physical exercise, breathing strategies, patient education and anxiety management $(30,33)$.

Aerobic exercises consisting primarily of supervised indoor and outdoor walking or stationary cycling, increase the functioning of immune system, too $(3,8)$. All patients should be encouraged to take part in this routine every day (30). Physical exercises must alternate with pauses - the target saturation being $\mathrm{SpO} 2>90 \%$ and oxygen supplementation is recommended during training in order to unload the respiratory muscles $(30,34)$.

Early pulmonary rehabilitation in ICU for COVID-19 inpatients should be done graded, pointing out the breathing exercises and the bed mobility (i.e. flexion/extension of legs, arms stretches, sitting/standing at bedside) and having in mind the fact that $3 \%$ to $5 \%$ of patients can progress within 7 to 14 days of infection to severe or critical state (28). Respiratory rehabilitation is not recommended for severely and critically ill patients until their condition becomes stabilized. In patients needing prolonged mechanical ventilation, inspiratory and expiratory muscles training is a path to follow, using the respiratory adequate devices (30).
Starting with June of 2020, a team consisting of a physical medicine and rehabilitation physician and four physiotherapists from "Dr. Nicolae Robanescu" National Clinical Teaching Hospital for Pediatric Neuro-Rehabilitation have been detached to assist, evaluate and contribute to COVID-19 critical ill patients' rehabilitation in Intensive Care Units (ICU) and clinical wards of "Matei Bals" National Institute of Infectious Disease.

As for October 2020 and still ongoing, a number of approximately fifty patients from both the ICU and clinical wards have been evaluated by the physician and underwent a physical therapy program, assisted by the physiotherapist.

In the ICU, the main challenges consist in managing the critical ill patients that are mechanically ventilated and sedated, due to the numerous complications of SARS-Cov2 infection that often require vasopressor assistance and hemodialysis, factors which limit the human resource in applying physiotherapy techniques.

To the hemodynamic stable and intubated patients, our main objectives are to apply the basic kinesiotherapy principles to maintain the neurological cortical pathways and prevent the long term complications which are frequently encountered in bedridden patients, as well as preparing the sedated patients for a better recovery after extubation. Also, bed posturing is an essential technique in respiratory rehabilitation, as prone position is preferred for better ventilation, up for sixteen hours a day for each patient along the modified Trendelenburg position. In such patients, the proprioceptive focal vibration device was applied in order to maintain the physiological gait pathway, during sedation.

The ICU conscious patients that require high flow oxygen administration and present persistent irritative dry cough benefit from same neuro-muscular techniques among respiratory rehabilitation procedures that consist of postural drainage therapy, controlled cough maneuvers, sputum drainage procedures, adjusting chest expansion for better inspiratory inflow. The ventilation positions are also recommended during the days and especially during sleeping hours.

The upper mentioned techniques are often interchangeable, as ICU patients' clinical evolution is most often unpredictable, in numerous cases exitus being an outcome.

To the patients that survive COVID-19 complications and are discharged from the ICU to the clinical wards, it is important to be followed up by the rehabilitation team, in order to continue the 
program, as the whole medical team has noticed a better outcome in respiratory levels and deconditioning delaying.

In clinical wards, were patients are awake and some require medium to low levels of oxygen through facial masks, the intensity of the muscular and respiratory training is adapted to each individual's clinical presentation, muscle strength and associated comorbidities that may impair the patient.

In these situations, the respiratory rehabilitation program is better applied as the patients willingly contribute to the assisted techniques. Breathing retraining, cough assisting, bronchial drainage, improving the respiratory muscles' tonus are quintessential for a better outcome.

For most cases, we encourage the patients to watch the monitors and follow the saturation levels, as the increment in this parameter often leads to a better state of mind and wish to continue the respiratory recovery process. Also, the patients are recommended to use the prone position during sleeping hours and lateral decubitus during the day, to expand each lung during breathing and thus improving the respiratory parameters.

So far, it is known that the main complications of COVID-19 pneumonia are the long lasting pulmonary fibrin lesions which in time lead to restrictive respiratory dysfunction with oxygen requirement, altering the individual's life, leading to psychological distress also. Per se, the illness is associated with anxiety, sleeping disorders, cognitive impairment, besides the well-known symptoms, situations which delay clinical and physical rehabilitation and extend hospitalization days. Long term hospitalization and the consumption nature of the disease lead to muscular atrophy. All the previous complications are better handled and fought off through adapted exercises, practiced by the physiotherapists.

Early intervention and implementation of the rehabilitation program leads to a better long term outcome for both intubated patients and non-intubated ones, as it prevents deconditioning and improves vital parameters, as we observed during these past four months.

From the physician's point of view, it is extremely important to carefully conduct the anamnestic process and evaluation, to adjust the program to each individual, to monitor oxygen saturation, blood pressure levels, pulse and temperature throughout the exercises. Every increment or decrement of these parameters, onset of cough or fatigability, must end the rehabilitation process.
Discharge recommendations consist in promoting a healthy lifestyle through weight control, practicing aerobic exercises with overtime intensity adjustment (from fast walking, to bicycling, swimming, jogging) and most importantly, continuing the learnt respiratory exercises with a daily frequency, to maintain ventilator positions during bed rest.

As a challenge for both the physician and physiotherapist is represented by the personal protection equipment, making it hard to conduct the evaluation and rehabilitation process, due to the constant heat exposure and low intake of oxygen as well as difficulties in managing and handling the patient.

\section{DISCUSSIONS}

Overall, the main targets of respiratory rehabilitation are (35):

- Maintaining the flow in the respiratory tract using positioning, mobilization, cough techniques

- Reducing the dyspnea

- Maintaining the optimal functioning of the diaphragm and of accessory muscles

- Improving chest mobility by correcting the deformities of the spine using appropriate kinetotherapy

- Increasing the functional exercise capacity

- Alleviating both anxiety and/ or depression

- Improving the functional status and the quality of life as much as possible

- Diminishing morbidity

The rehabilitation programme should be adjusted also to the existing co-morbididies: hypertension, diabetes mellitus, neuromuscular diseases - significant risk factors for developing severe forms of COVID-19. In these unhappy situations, it is important to maintain the heart functional condition, joint flexibility, muscle strength, pulmonary rehabilitation having a main role including in the medical assistive process (3).

Obesity is another risk factor for sever evolution of COVID-19, especially in obesity with BMI-41 (30) and the nutrition promotion is required. These patients have to be motivated to change their food style and information on appropriate diet and exercise will surely reinforce habit change.

It should be mentioned, in an extremely recent study, as conclusions from the retrospective analysis of a research comprising 119 patients hospitalized for COVID-19, the following are stipulated: "Interestingly, pre-existing conditions including hypertension, severe obesity, lung disease, 
and diabetes did not correlate with dependent functional status at discharge" (36).

In cases with severe functional impairments, with limited physical effort capability in activity of daily living (ADL), occupational and physical therapy may also be considered (30). Individual inhalation therapy (although there are some debates in the literature regarding the procedures generating aerosols (37)), speleotherapy and forest cure are recommended as well in pulmonary rehabilitation in many balnear resorts $(3,38)$.

In the subchronic-chronic stages, with respect to all epidemiological precautions both regarding the triage for hospitalization of post-COVID-19 patients and the sanitary norms of operation in the current pandemic of the medical-spa units with respiratory profile, could make a beneficial contribution as a later stage - or even, hopefully, the final - in the complete rehabilitation of the postCOVID-19 sequelae (39).

Pulmonary rehabilitation for COVID-19 patients has become a major challenge for medical staff, giving us the additional support to fight against SARS-CoV-2 and should be provided both in hospitalized or at home patients. Using an individualised assessment and methods, is becomes a great tool for returning the individuals in society, helping them to maximise their functioning and consequently their quality of life (40).

\section{REFERENCES}

1. https://bestpractice.bmj.com/topics/en-gb/3000201.

2. Worobey M, Pekar J, LarsenB, Nelson M, Hill V, Joy J, Rambaut A, Suchard M, Wertheim J, Lemey P. The emergence of SARS-CoV-2 in Europe and North America. Science 2020; eabc8169.

3. Munteanu C, Paun DL, Șuță AM, Florescu SA, Onose G. Diabetus mellitus and COVID-19 in the post-acute phase patients - possible links with physical and rehabilitation medicine and balneotherapy. Balneo Research Journal 2010;11(3).

4. https://www.who.int/dg/speeches/detail/who-director-general-sopening-remarks-at-the-media-briefing-on-covid-19---11-march-2020.

5. Tabata S, Imai K, Kawano S, Ikeda M, Kodama T, Miyoshi K et al. Clinical characteristics of COVID-19 in 104 people with SARS-CoV-2 infection on the Diamond Printess cruise ship: a retrospective analyse. The Lancet, 2020;20(9).

6. Barupal T, Tak PK, Meena M. COVID-19: Morphology, Characteristics, Symptoms, Prevention, Clinical Diagnosis and Current Scenario. Coronaviruses, 2020;1(1).

7. Leung NH, Chu DK, Shiu EY et al. Respiratory virus shedding in exhaled breath and efficacy of face masks. Nat Med. 2020.

8. https://www.cdc.gov/coronavirus/2019-ncov/prevent-getting-sick/ how-covid-spreads.html.

9. Hermann M, Pekacka-Egli A-M, Witassek F, Baumgaertner R, Schoendorf S, Spielmanns M. Feasibility and Efficacy of Cardiopulmonary Rehabilitation After COVID-19. American Journal of Physical Medicine \& Rehabilitation 2020;99(10).

\section{CONCLUSIONS}

Based on both the necessary theoretic documentation and respectively, the lessons learned from the experience related to pulmonary rehabilitation in COVID-19/post COVID-19 patients we reckon this is important for further improving our capabilities to help these patients and also for a better holistic management of such cases even, if necessary, later on, subsequent to having been cured after overcoming the acute/critical stage.

\section{Acknowledgements}

All authors listed have made consistent contributions on various specific levels overall, in equivalent parts.

This work has received the approval of the Ethics Commission of the "Prof. Dr. Matei Bals" National Institute of Infectious Diseases in Bucharest (number 11324/16.10.2020), "Dr. Nicolae Robanescu" National Clinical Teaching Centre for Pediatric Neuropsychomotor Rehabilitation in $\mathrm{Bu}-$ charest (number 7026/13.10.2020), "Bagdasar-Arseni" Teaching Emergency Hospital in Bucharest (number 34996/ 13.10.2020), Romania.

\section{Disclaimer}

This article, in its Romanian version, has been invited to be published (also) in Romanian Medical Journal 2020;67(Suppl. 6).

Conflict of interest: none declared Financial support: none declared

10. Varga Z, Flammer AJ, Steiger P et al. Endothelial cell infection and endotheliitis in COVID-19. Lancet 2020;395:1417-8.

11. Guzik TJ Mohiddin SA Dimarco A et al. COVID-19 and the cardiovascular system: implications for risk assessment, diagnosis, and treatment options. Cardiovasc Res. 2020.

12. Levi M, Thachil J, Iba T, Levi JH. Coagulation abnormalities and thrombosis in patients with COVID-19. The Lancet Haemathology 2020;7(6):E438-E440.

13. Dick EC, Jennings LC, Mink KA, Wartgow CD, Inhorn SL. Aerosol transmission of rhinovirus colds. J Infect Dis. 1987;156:442-8.

14. Wong TW, Lee CK, Tam W, Lau JT, Yu TS, Lui SF et al. Cluster of SARS among medical students exposed to single patient, Hong Kong. Emerg Infect Dis. 2004;10:269-76.

15. Galbadage T, Peterson BM, Gunasekera R. Does COVID-19 spread through droplets alone? Front Public Health. 2020;8:163.

16. University of Maryland. Wearing surgical masks in public could help slow COVID-19 pandemic's advance: masks may limit the spread diseases including influenza, rhinoviruses and coronaviruses. https:// www.sciencedaily.com/releases/2020/04/200403132345.htm.

17. Migliori GB, Nardell E, Yedilbayev A et al. Reducing tuberculosis transmission: a consensus document from the World Health Organization Regional Office for Europe. European Respiratory Journal 2019;53:1900391.

18. Esposito S, Principi N, Leung CC, Migliori GB. Universal use of face masks for success against COVID-19: evidence and implications for prevention policies. European Respiratory Journal 2020;55:2001260. 
19. Fraser E. Long term respiratory complications of COVID-19. BMJ. 2020;370:m3001.

20. Asthma UK, British Lung Foundation. Post-COVID hub. https://www. post-covid.org.uk/get-support/.

21. https://www.thelancet.com/journals/lancet/article/PIIS01406736(20)31182-X/fulltext.

22. Spagnolo P, Balestro E, Aliberti S, Cocconcelli E et al. Pulmonary fibrosis secondary to COVID-19:a call to arms? Lancet Respir Med. 2020;8(8)

23. Parshely L. The emerging long-term complications of COVID-19, explained. The vox guide to navigating the coronavirus crisis, Jun 2020.

24. Rubin GD, Ryerson CJ, Haramati LB, Sverzellati N, Kanne JP, Raoof $S$ et al. The Role of Chest Imaging in Patient Management during the COVID-19 Pandemic: A Multinational Consensus Statement from the Fleischner Society. Radiology. 2020; 201365.

25. Beusekom VM. Another possible COVID complication: "punctured lung". CIDRAP. 2020.

26. Xu Z, Shi L, Wang Y, Zhang J, Huang L, Zhang C, Liu S, Zhao P, Liu H, Zhu L, Tai Y, Bai C, Gao T, Song J, Xia P, Dong J, Zhao J, Wang FS. Pathological findings of COVID-19 associated with acute respiratory distress syndrome. Lancet Respir Med. 2020; 8(4):420-422.

27. Gomersall CD, Joynt GM, Lam P, Li T, Yap F, Lam D, Buckley TA, Sung JJ, Hui DS, Antonio GE, Ahuja AT, Leung P. Short-term outcome of critically ill patients with severe acute respiratory syndrome. Intensive Care Med. 2004;30(3):381-387.

28. Consoli L, Bendotti V, Cicchinelli S, Gaioni F, Prandolini P, et al. 2019 novel coronavirus (COVID-19) pneumonia complications: the importance of lung ultrasound. J Ultrasound. Jun 2020.

29. Zhao YM, Shang YM, Song WB, Li QQ, Xie H et al. Follow-up study of the pulmonary function and related physiological characteristics of COVID-19 survivors three months after recovery. The Lancet. 2020.
30. Wang TJ, Chau B, Lui M, Lam G-T, Lin N, Humbert S et al. PM\&R and Pulmonary Rehabilitation for COVID-19. Am J Phys Med Rehabil. 2020

31. Bailey P, Thomsen GE, Spuhler VJ, Blair R, Jewkes J, Bezdjian L et al. Early activity is feasible and safe in respiratory failure patients. Crit Care Med. 2007;35(1):139-45.

32. Poletti V, Capozzolo A. Respiratory rehabilitation in COVID-19 era. Respiration 2020;99:461-462.

33. Chinese Association of Rehabilitation Medicine, Respiratory rehabilitation committee of Chinese Association of Rehabilitation Medicine, Cardiopulmonary rehabilitation Group of Chinese Society of Physicai Medicine and Rehabilitation [Recommendations for respiratory rehabilitation of COVID-19 in adult]. Zhonghua Jie $\mathrm{He} \mathrm{He}$ Hu Xi Za Zhi. 2020;43:E029.

34. Lim PA, Ng YS, Tay BK. Impact of a viral respiratory epidemic on the practice of medicine and rehabilitation: severe acute respiratory syndrome. Arch Phys Med Rehabil. 2004;85:1365-1370.

35. Aytur YK, Koseoglu BF, Taskiran OO, Ordu-Gokkaya NK, Delialioglu $\mathrm{SL}$, et al. Pulmonary rehabilitation principles in SARS-CoV-2 infection (COVID-19): a guideline for acute and subacute rehabilitation. Turk $J$ Phys Med Rehabil. 2020;66(2):104-120.

36. Leigh AE, McCall J, Burke RV, Rome R., Raines AM. Predictors of functional dependence after COVID-19. American Journal of Physical Medicine \& Rehabilitation. 2020.

37. Bolton L, Mills C, Wallace S, Brady MC. Aerosol generating procedures, dysphagia assessment and COVID-19: A rapid review. International Journal of Language \& Communication Disorders. 2020; 55(4):629-636.

38. Sbenghe T. Recuperarea medicală a bolnavilor respiratori. Ed Medicală, 1983

39. http://legislatie.just.ro/Public/DetaliiDocument/226769.

40. Sheehy LM. Considerations for Postacute Rehabilitation for Survivors of COVID-19. JMIR Public Health Surveill. 2020;6(2):e19462. 